\title{
Non equilibrium molecular dynamics simulations of the thermal conductivity of water: a systematic investigation of the SPC/E and TIP4P/2005 models
}

\author{
Frank Römer
}

Chemical Physics Section, Department of Chemistry, Imperial College London, $S W^{\text {r }}$ 2AZ, London $U K$

Anders Lervik

Department of Chemistry, Norwegian University of Science and Technology, Trondheim, Norway

Fernando Bresme ${ }^{\mathrm{a})}$

Chemical Physics Section, Department of Chemistry, Imperial College London,

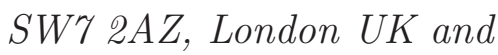

Department of Chemistry, Norwegian University of Science and Technology, Trondheim, Norway

(Dated: 12 July 2012)

We report an extensive non equilibrium molecular dynamics investigation of the thermal conductivity of water using two of the most accurate rigid non polarizable empirical models available, SPC/E and TIP4P/2005. Our study covers liquid and supercritical states. Both models predict the anomalous increase of the thermal conductivity with temperature and the thermal conductivity maximum, hence confirming their ability to reproduce the complex anomalous behaviour of water. The performance of the models strongly depends on the thermodynamic state investigated, and best agreement with experiment is obtained for states close to the liquid coexistence line and at high densities and temperatures. Considering the simplicity of these two models the overall agreement with experiments is remarkable. Our results show that explicit polarizability and molecular flexibility are not needed to reproduce the anomalous heat conduction of water.

a)Electronic mail: f.bresme@imperial.ac.uk 


\section{INTRODUCTION}

The understanding of heat conduction in fluids is a problem of great relevance in heat management applications. With the miniaturisation of electronic components, with dimensions of tens of nanometers, scientists and engineers face new challenges in the design of fluids that can efficiently transport the dissipated heat. Hence, it is important to understand the microscopic mechanisms determining the heat transfer of fluids, and how the transfer depends on the presence of additives. By defining suitable interaction potentials, computer simulations provide a unique approach to address these questions from a microscopic perspective. It is possible to estimate transport coefficients, in particular the thermal conductivity, using the Green Kubo approach ${ }^{1}$, or by computing the fluid response to an explicit temperature gradient ${ }^{2-6}$. Simulations thus provide a route to perform systematic investigations of the thermal conductivity in a variety of thermodynamic conditions. It is then possible to rationalize the performance of fluid suspensions (nanofluids) in heat management problems $^{7-9}$, or to quantify heat transfer in nanomaterials ${ }^{10}$ and biological assemblies. The latter is a problem of particular significance in chemical physics and biophysics ${ }^{11,12}$.

In this article we report a comprehensive investigation of the thermal conductivity of water. Water plays an important role in thermal management applications, since it has the highest thermal conductivity observed in any molecular liquid. The investigation of heat transfer in water is also a problem of great fundamental interest, as the thermal conductivity features an anomalous increase with temperature and a maximum. To investigate heat conduction in water we employ boundary driven non equilibrium molecular dynamics simulations (NEMD), in combination with the most accurate empirical force fields available for water, namely, the SPC/E $\mathrm{E}^{13}$ and TIP4P $/ 2005^{14}$. The equilibrium and coexistence properties of these two models have been extensively investigated. The review by Abascal and Vega $^{15}$ is of particular significance in this context. They reported a detailed and critical discussion of the accuracy of the most popular rigid and non polarizable water models. The main conclusion from this analysis is that SPC/E and TIP4P/2005 are the most accurate three site and four site models respectively. In terms of overall accuracy, TIP4P/2005 is superior, providing good estimates of the structure of water, equation of state, liquid vapour coexistence curve, surface tensions and dynamic properties. Following the trends of other empirical models, the TIP4P/2005 potential underestimates the vapour pressure as well 
as the dielectric constant. These discrepancies are expected, as the models does not include molecular polarizability. Despite these shortcomings, the accuracy of the TIP4P/2005 potential is remarkable, and it remains the best choice for the investigation of water and aqueous solutions that require a large number of molecules. Part of the success of this model is connected to the implementation of an off site negative charge, which leads to a better prediction of the ratio of dipole and quadrupole moments of the water molecule ${ }^{16}$.

The thermal conductivity of water has been computed before using a variety of techniques and models ${ }^{17-22}$. Most of these studies have focused on a specific temperature and density or a narrow range of thermodynamic states. The general conclusion from these studies is that the thermal conductivity is overestimated, typically by about $20 \%$, at temperatures close to $300 \mathrm{~K}$ and 1 bar pressure. Some of these studies have given an indication that the models can reproduce the anomalous increase of the thermal conductivity with temperature ${ }^{18,21,22}$. Recent simulations using the TIP5P model have also predicted the existence of a minimum in the thermal conductivity in the supercooled region ${ }^{23}$, which would be connected to the proximity of a possible liquid-liquid transition that has been predicted using of computer simulations. Given the limited number of studies and thermodynamic states, it is not possible to draw general conclusions regarding the ability of empirical models to predict heat transfer in water. In this paper we significantly extend these investigations by performing non equilibrium simulations, covering a wide range of thermodynamic states, including liquid and supercritical states, and densities below and above the critical one.

The paper is structured as follows. We first describe the simulation details and the non equilibrium approach employed in this paper. A discussion of the main results, the equations of state obtained via non equilibrium simulations and thermal conductivities follows. The main conclusions and final comments close the paper.

\section{COMPUTER SIMULATIONS}

The simulations were performed using orthorhombic periodic cells with dimensions $\left\{L_{x}, L_{y}, L_{z}\right\} / L_{x}=\{1,1,3\}$ with $L_{x}$ in the interval $3.778 \mathrm{~nm}$ and $6.46 \mathrm{~nm}$ depending on the average density of the system. The simulation box contained 5410 water molecules. We

employed the leap-frog ${ }^{24}$ algorithm with a time step of 2 fs for the integration of Newton's equations of motion. Averages were written every 1000 steps in order to perform further 
analyses. The short range Lennard-Jones (LJ) interactions acting between the oxygen sites, were truncated at a cut-off distance of $r_{\mathrm{c}}=1.9 \mathrm{~nm}$, corresponding to $\sim 6 \sigma_{\mathrm{O}}$, where $\sigma_{\mathrm{O}}$ is the effective diameter of the water molecule in the TIP4P/2005 and SPC/E models. This cut-off is long enough to reproduce the properties of the full potential, hence, no long range corrections were considered. It has been noted that the thermal conductivity slightly depends on whether long range coulombic interactions are included or not ${ }^{22}$. To address this issue, coulomb interactions were computed using the Particle-Mesh ${ }^{25}$ (PME) variant of the Ewald method, with a mesh width of $0.12 \mathrm{~nm}$ and an interpolation order of four. The real space cut-off was the same as that employed for the LJ potential. The potential parameters for TIP4P/2005 and SPC/E were taken from the original publications ${ }^{13,14}$. The molecules were maintained rigid by using the $\mathrm{SETTLE}^{26}$ algorithm.

The non equilibrium computations were performed using boundary driven molecular dynamics simulations $s^{2,3,18}$. In this approach we define hot and cold thermostats at the edges and in the middle of the box respectively. The configurations were generated as follows. Firstly, a simulation box containing water molecules was simulated in the canonical ensemble $(N V T)$ for 200 ps at a selected temperature and density. We employed the velocity rescale $\operatorname{algorithm}^{27}$ to thermalize the system at $T_{\text {ave }}=\left(T_{\text {hot }}+T_{\text {cold }}\right) / 2$, where $T_{\text {hot }}$ and $T_{\text {cold }}$ are the desired temperatures for the hot and cold thermostats respectively. The thickness of the thermostatting layers was between $0.09-0.16 \mathrm{~nm}$. These layers contained about $3 \%$ of the total molecules in the simulation box. For an equilibrated configuration, the positions of the oxygen atoms lying inside the pre-defined hot and cold layers were restrained using harmonic potentials with a force constant of $1 \mathrm{MJ} \mathrm{mol}^{-1} \mathrm{~nm}^{-2}$. Because we "restrain" the position of the oxygens only, the molecules can rotate freely. These "hot" and "cold" molecules were then thermostated to the desired temperatures, $T_{\text {hot }}$ and $T_{\text {cold }}$. Several approaches can be used with this purpose. In this work we used the velocity rescale algorithm ${ }^{27}$, which samples the canonical distribution by appropriately rescaling the velocities using a stochastic scaling factor. We considered a time coupling constant of $\tau=0$ ps. For this time constant, the velocity rescale algorithm simplifies to the stochastic velocity rescaling approach, where the factor used to rescale the velocities is given by,

$$
\alpha=\sqrt{\frac{K_{t}}{K}}
$$

where $K$ is the instantaneous kinetic energy of the system, and $K_{t}$ is the target value of the 
kinetic energy. This kinetic energy, $K_{t}$, is drawn from the canonical distribution function ${ }^{27}$,

$$
\mathcal{P}\left(K_{t}\right) d K_{t} \propto K_{t}^{N_{f} / 2-1} e^{-\beta K_{t}} d K_{t}
$$

where $N_{f}$ is the number of degrees of freedom associated with the thermostatted molecules and $\beta=1 /\left(k_{B} T\right)$.

In our approach we do not thermostat the "unrestrained" molecules. Hence, any molecule entering the hot or cold region exchanges momentum with the thermostatted ("restrained") molecules, and will then become hotter or cooler. The thermostatting process generates a temperature gradient and a heat flux. The latter can be computed using the continuity equation,

$$
\mathbf{J}_{U}=\left[0,0, \pm \frac{\langle\Delta U\rangle}{2 \delta t A}\right]
$$

where, $A$ is the cross-sectional area perpendicular to the direction of the flux, $\delta t$ is the simulation time step and $\langle\Delta U\rangle$ the average change in internal energy in the cold (-) and hot $(+)$ layers. The brackets denote an ensemble average. By definition,

$$
\Delta U=\Delta V(\mathbf{r})+\Delta K(m, \mathbf{v})
$$

where $\Delta V(\mathbf{r})=V(\mathbf{r})_{a}-V(\mathbf{r})_{b}$ is the change in potential energy before and after the molecules have been thermostated. This change in potential energy is strictly zero, as the thermostatting process does not modify the configuration coordinates, r. Similarly,

$$
\Delta K(m, \mathbf{v})=K(m, \mathbf{v})_{a}-K(m, \mathbf{v})_{b}
$$

is the corresponding change in kinetic energy with

$$
K(m, \mathbf{v})=\frac{1}{2} \sum_{i=1}^{N \in T L} m_{i} v_{i}^{2} .
$$

The sum in the equation above runs over the particles in the corresponding thermostatting layer, $T L$. The change in kinetic energy, defines the change in internal energy, $\Delta U \equiv$ $\Delta K(m, \mathbf{v})$ that enters the continuity equation (3).

A typical simulation spanned $1 \mathrm{~ns}$, to ensure the stationary state was reached ${ }^{22}$. We then performed simulations for a further $1 \mathrm{~ns}$ in order to collect averages. The density 
and temperature profiles were computed locally, by dividing the simulation box in layers (typically 120), along the direction of the temperature gradient (the $z$-axis). The simulations were performed with a modified version of the GROMACS $\operatorname{code}^{28}$.

\section{RESULTS}

We have investigated 17 different thermodynamic state points, which cover liquid and super critical states and a wide range of densities. The average temperature in the simulation box ranged from $325 \mathrm{~K}$ to $725 \mathrm{~K}$ and the average density from $0.2 \mathrm{~g} \mathrm{~cm}^{-3}$ to $1.0 \mathrm{~g} \mathrm{~cm}^{-3}$. We first compare the accuracy of the water models in predicting the equation of state of water for the thermodynamic states investigated in this work. In figure (Fig. 1) we compare the equations of state computed using equilibrium molecular dynamics simulations in the isothermal-isobaric ensemble ( $N P T)$ with the isothermal and isochoric reference data from the National Institute of Standards and Technology database (NIST) ${ }^{29}$. Both, SPC/E and TIP4P/2005 models show good agreement with the reference data. At super critical temperatures both models slightly underestimate the pressure at low densities, and slightly overestimate it at high densities. The SPC/E provides a slightly better agreement with the NIST data at high temperatures, $\sim 700 \mathrm{~K}$ and low densities $<0.3 \mathrm{~g} / \mathrm{cm}^{3}$. In general the TIP4P/2005 potential shows a better agreement with the reference data in the high density

regime. Our results are consistent with a recent published comparative study ${ }^{15}$ devoted to computer simulations of water models, and confirm the good accuracy of the models chosen in this work.

We have also computed the equation of state for water using NEMD simulations. The NEMD approach employed here ensures that mechanical stability is fulfilled upon reaching the stationary state, i.e., $\nabla \mathbf{P}=0$, making possible the estimation of the equation of state for a given isobar using a single simulation. The temperature gradient induces a density gradient in the system, whose magnitude is determined by the thermal expansion of the liquid. Assuming that local equilibrium is fulfilled, in the sense that the thermodynamic relationships are valid at any point in the system, so that they can be expressed in terms of quantities defined locally ${ }^{30}$, we can estimate the equation of state by representing temperature and density pairs along the temperature gradient. It is implicit in this analysis that the size of the local volume investigated is large enough to converge to the bulk results. This 


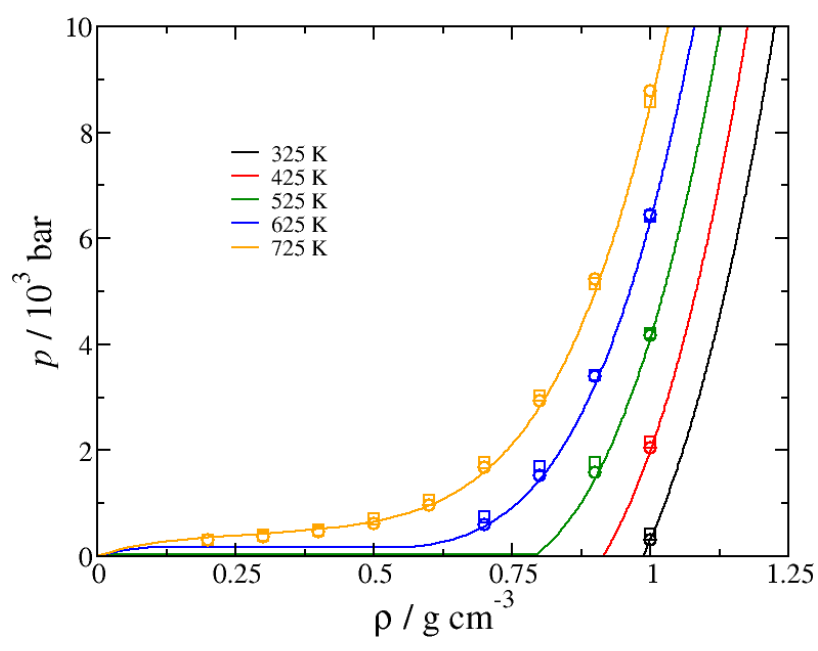

(a)

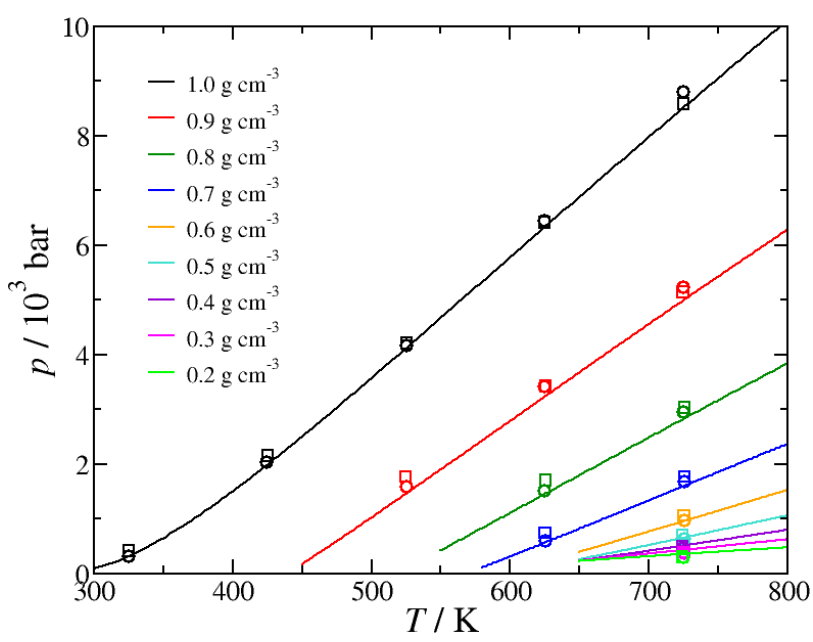

(b)

FIG. 1. Pressure (a) isotherms and (b) isochores of water. The points represent NPT equilibrium molecular dynamics simulations of $(\square)$ SPC/E and (o) TIP4P/2005 models. The lines represent data taken from the NIST database ${ }^{29}$.

hypothesis can be tested a posteriori by comparing the non equilibrium and equilibrium results for the equation of state. Our NEMD method generates two gradients in opposite direction, which are symmetric around the center of the simulation box. We took advantage of this fact to obtain a single average using the data from both sides of the box. For the analysis of the equation of state we discarded the layers near the thermostats, and we calculated the density using 15 sub-volumes in order to reduce the noise in the density that would appear otherwise from sampling very small subvolumes. Fig. 2 shows the equation of state obtained in this way. To test the NEMD results, we have performed equilibrium simulations in the NPT ensemble using 2180 water molecules and the same cut-off that we employed in the NEMD simulations. The densities form the equilibrium simulations were obtained from averages over 1 ns trajectories. We find an excellent agreement between the NEMD and EMD simulation results, confirming the advantage of the NEMD approach to construct isobars from a single simulation. The the agreement between the NEMD / EMD results and the NIST data verify the excellent performance of the TIP4P/2005 model (see Figure 2).

We have taken advantage of the NEMD simulations to explore the performance of the TIP4P/2005 for a wider range of thermodynamic conditions. Our results are reported in Figure 2-b. Again we note that the 6 isobars represented in that figure were obtained from 
(a)
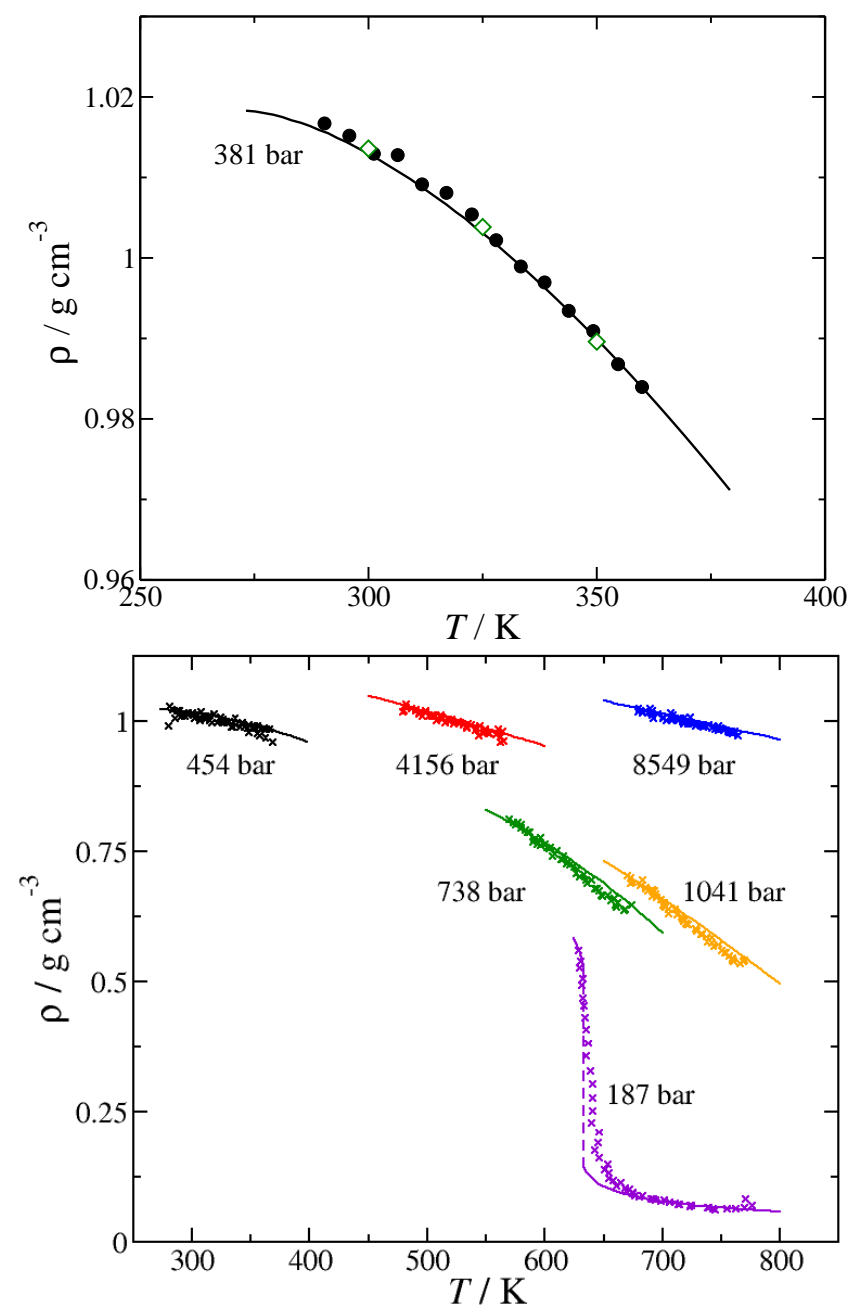

(b)

FIG. 2. Equation of state of water obtained from NEMD simulations: (a) Comparison of NEMD simulations of TIP4P/2005 (•) with the NIST database $(-)$. ( $\diamond)$ TIP4P/2005 results obtained from NPT equilibrium molecular dynamics simulations. (b) (×) TIP4P/2005 data obtained from non equilibrium molecular dynamics simulations, and $(-)$ data from the NIST database.

6 separate NEMD simulations. In this case we calculated the densities using 50 layers, discarding 5 layers next the hot and cold thermostats. The agreement between the TIP4P/2005 model and the experiments is excellent. We find some deviations at higher pressures and temperatures, as well as in the critical region (see the 187 bar isobar in Figure 2-b). In this later case the NEMD isobar has the characteristic shape of a supercritical state that is not far from the critical point. The discrepancies between the TIP4P/2005 and experimental data at this near critical isobar are expected as it is known that the TIP4P/2005 model 
predicts a too low critical pressure, only 146 bars, versus 220.6 bar observed in experiments.

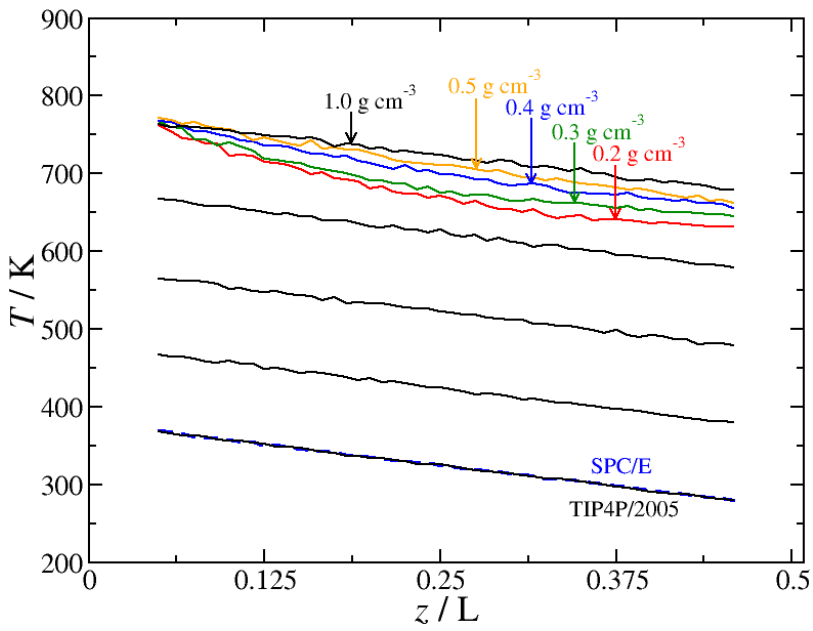

(a)

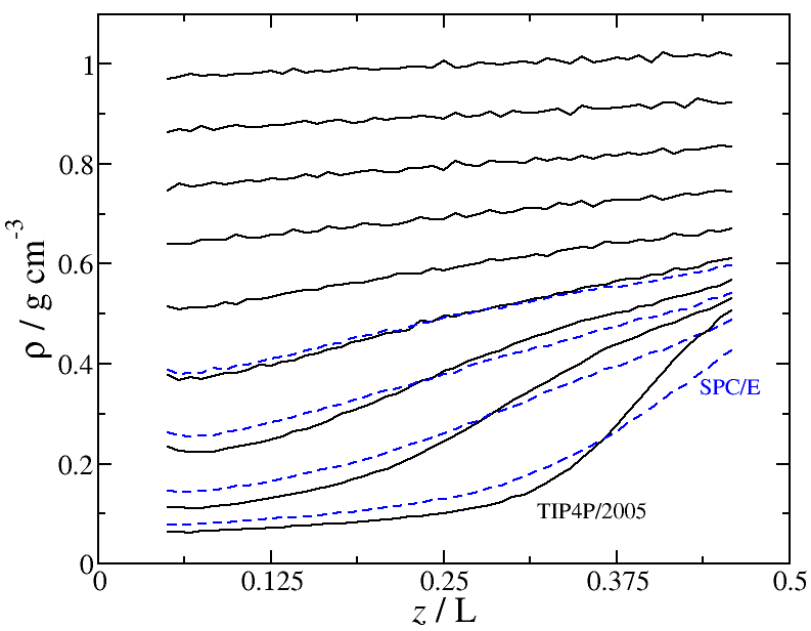

(b)

FIG. 3. (a) Temperature profiles along the simulation box for the TIP4P/2005 model and for different average temperatures at an average density of $1 \mathrm{~g} / \mathrm{cm}^{3}$ (black lines). For $T_{\text {ave }}=725 \mathrm{~K}$, the lines correspond to $\rho_{\text {ave }} /\left(\mathrm{g} / \mathrm{cm}^{3}\right)=0.5$ (orange), 0.4 (blue), 0.3 (green) and 0.2 (red line). Results for the $\mathrm{SPC} / \mathrm{E}$ model (dashed, blue line) at $T_{\mathrm{ave}}=325 \mathrm{~K}$ are also shown. (b) Density profiles along the simulation box for TIP4P/2005 (black line) and SPC/E (dashed, blue line). The latter at $T_{\text {ave }}=725 \mathrm{~K}$.

Having established the accuracy of the models in reproducing the equation of state for the thermodynamic states we are interested in, we focus in the following on the thermal conductivity. The thermal conductivity can be computed from Fourier's law,

$$
\mathbf{J}_{q} \equiv \mathbf{J}_{U}=-\lambda \nabla T
$$

where we identify the heat flux, $\mathbf{J}_{q}$ in the direction of the gradient, $z$, with the internal energy flux, $\mathbf{J}_{U}$, as there is no mass flux in the system. The internal energy flux is computed from equation (3) as discussed in the methodology section. In order to use equation (7) we need to calculate the temperature gradient, $\nabla T$. Figure 3 shows representative temperature profiles obtained in our simulations for a variety of thermodynamic states. It is important to note that the gradients do not need to be linear, and in fact they will not be linear in general. We expect that the local slope of the gradient will reflect changes in the local thermal conductivity of the fluid. This issue has been pointed out in a previous work ${ }^{21}$. 
The deviation from linearity is particularly clear at high temperatures, in the vicinity of the critical point (see data for $T>600 \mathrm{~K}$ in Figure 3). This behavior can be understood by inspecting the corresponding density profile (see Figure 3). The deviation from linearity of our temperature profiles is consistent with the rapid variation in density undergone by the fluid when the NEMD simulations drives the system to thermodynamic states near the critical point. We find that both SPC/E and TIP4P/2005 feature similar changes regarding the rapid variation of the density and deviation of the thermal gradients from the linear behaviour. A practical consequence of this lack of linearity is that the temperature gradient has to be computed with care in order to account for its local variations, as the thermal conductivity changes along the simulation box with temperature and density. We note that the non linear dependence of the temperature gradient does not imply a breakdown of Fourier's law. As noted above it just shows that a local temperature gradient must be considered to compute a local thermal conductivity. Hence, we splited the temperature profile in segments so that we could capture the local variations observed in the thermal gradients.

Figure 4 shows our first set of data for the thermal conductivity of SPC/E and TIP4P/2005 water for 17 different thermodynamic states. Our data at a density of $\rho=1 \mathrm{~g} / \mathrm{cm}^{3}$ are in good agreement with the few computations that have been reported before at $\sim 300$ K. The models overestimate the thermal conductivity for this thermodynamic state. This is a general feature observed in all computational studies performed to date with classical models, either rigid or central force fully flexible models ${ }^{18,21}$. The SPC/E and TIP4P/2005 models predict thermal conductivities that are identical within the statistical uncertainties of our computations, a conclusion that applies to all the 17 thermodynamic states shown in Figure 4. Numerical data for the thermal conductivity are compiled in tables I and II.

Our data provide a more comprehensive view on the performance of the water models. Based on the scattered data reported in previous works, the general conclusion would be that current models are fairly inaccurate. We find nonetheless that the agreement at $\rho=1$ $\mathrm{g} / \mathrm{cm}^{3}$ improves at higher temperatures, $T \sim 500 \mathrm{~K}$. For lower densities, in the range $0.9>\rho>0.5 \mathrm{~g} / \mathrm{cm}^{3}$ the agreement between the simulations and experiment is almost quantitative (see Figure 4). At lower densities we find again larger discrepancies that might be connected to the inaccuracy of the models in reproducing the properties of water in the low density regime. It is encouraging that all the water models investigated to date reproduce 


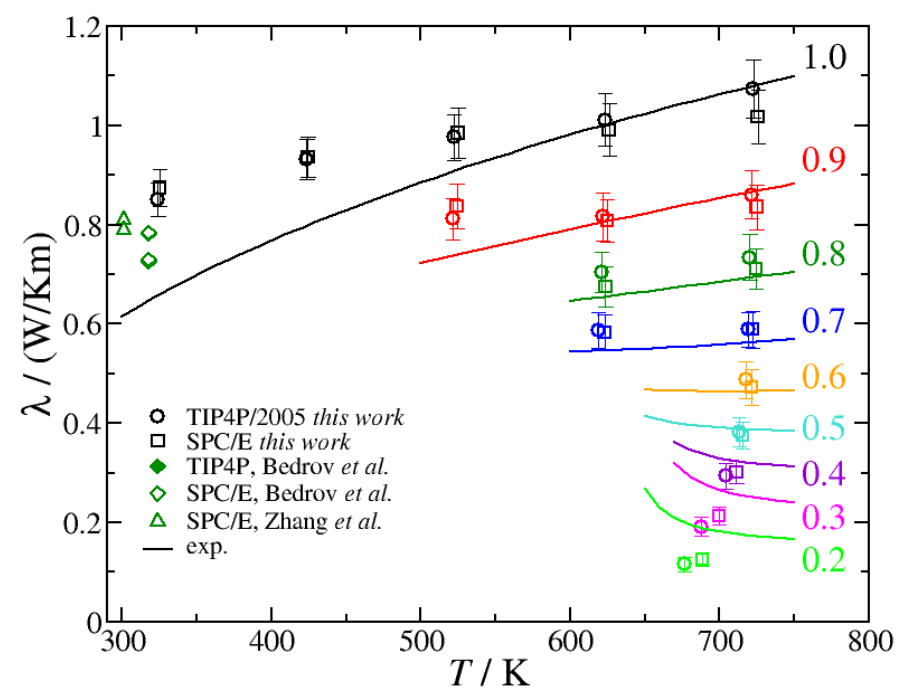

FIG. 4. Thermal conductivity versus temperature for (o) TIP4P/2005 and ( $\square$ ) SPC/E and at different densities (in $\mathrm{g} / \mathrm{cm}^{3}$ ). The solid lines represent experimental isochoric data from reference ${ }^{31}$. Diamonds represent simulation data from reference ${ }^{17}$ and triangles from reference ${ }^{19}$.

the anomalous increase in the thermal conductivity of water, which is clearly observed at higher densities (see $\rho>0.9 \mathrm{~g} / \mathrm{cm}^{3}$ isochores in Figure 4). To the best of our knowledge the first computational evidence for the anomalous increase in the thermal conductivity of water was reported using the Central Force Model $^{18,21}$.

Figures 5-a and b show the thermal conductivities of the TIP4P/2005 and SPC/E models investigated in this work. The data cover again liquid and supercritical states at different densities and pressures ranging from the supercritical pressure up to 5000 bars. We calculated the thermal conductivities by splitting the temperature gradient in segments, and the local thermal conductivity was then computed for each segment. Again, we discounted from this analysis 5 layers near the hot and cold thermostats. Both models, SPC/E and TIP4P/2005 predict similar results. We find nonetheless some differences in the accuracy of the models at low density and high temperature, where the SPC/E model is slightly more accurate. It is known that the $\mathrm{SPC} / \mathrm{E}$ equation of state is also slightly more accurate at low densities and subcritical temperatures. We showed above that it is also slightly more accurate at low densities and high temperatures (see Figure 1). This may influence the computation of the thermal conductivity. However, we note that the differences between the simulation values are within $\sim 10 \%$, and therefore close to the uncertainty associated 


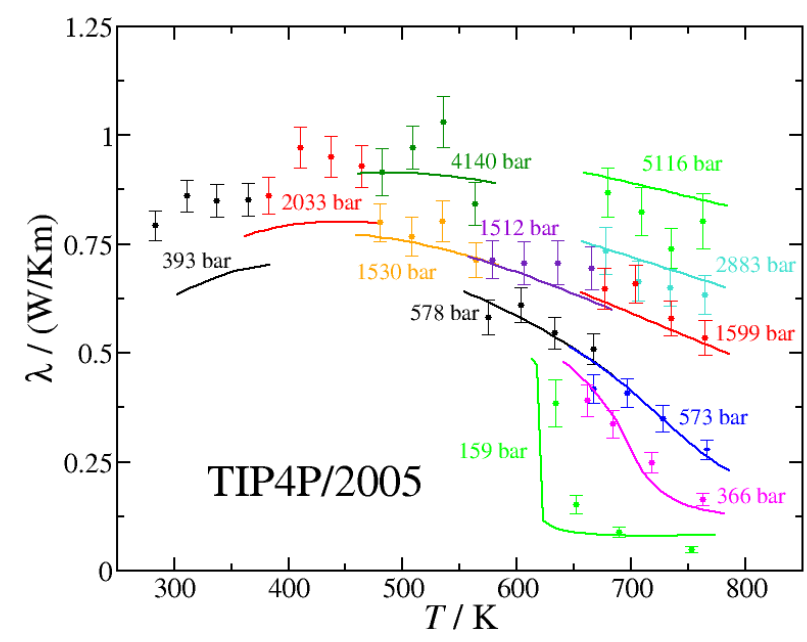

(a)

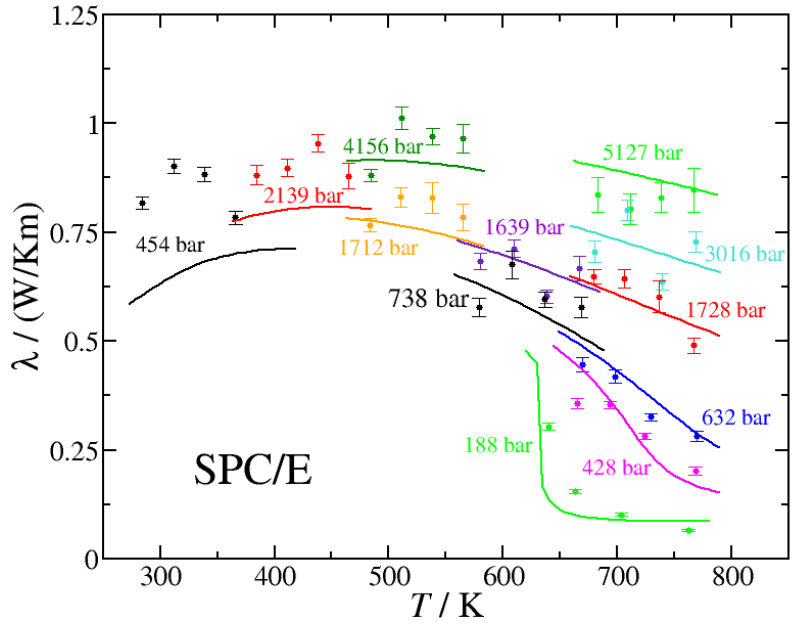

(b)

FIG. 5. Thermal conductivity versus temperature for (a) TIP4P/2005 and (b) SPC/E. The temperature and the thermal conductivity are calculated for each point by splitting the thermal gradient in different regions. See text for details. The solid lines correspond to data obtained from reference $^{31}$.

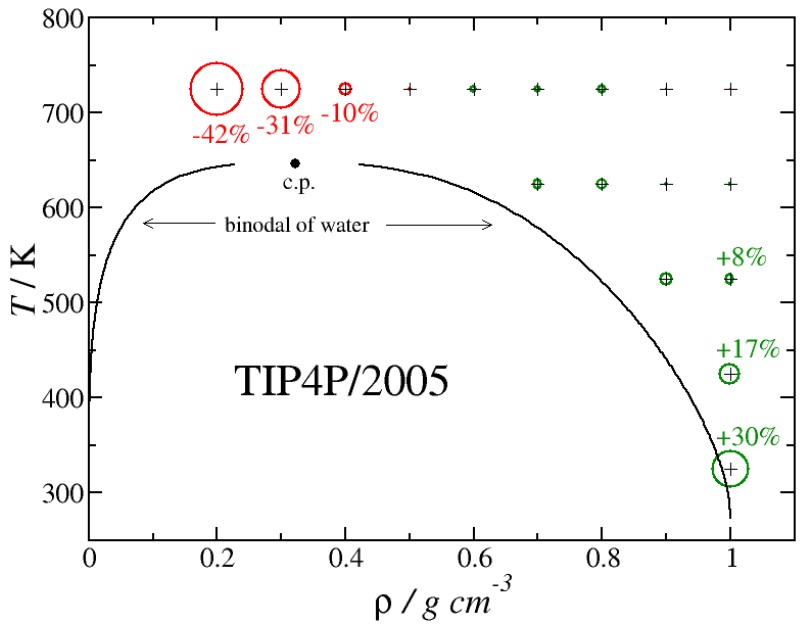

(a)

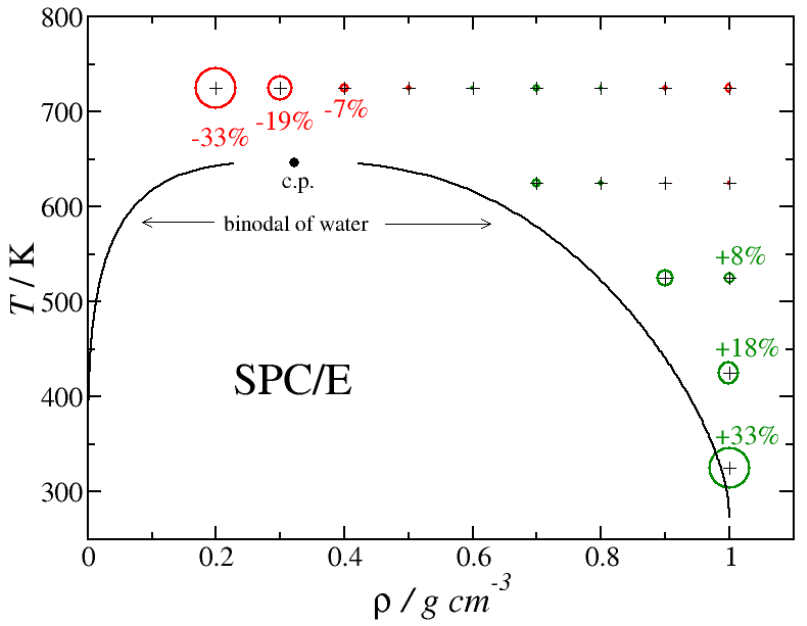

(b)

FIG. 6. Phase diagram of water: $(-)$ binodal and $(\bullet)$ critical point taken from $\mathrm{NIST}^{29}$. We have represented with crosses, $(+)$, the state points, i.e., average temperature and density, used in the NEMD simulations. The circles provide a visual representation of the relative error of the thermal conductivities predicted by the (a) TIP4P/2005 and (b) SPC/E models with respect to data from reference $^{31}$. 
to computations of the thermal conductivity. The ability of the models to predict the correct physical behaviour of the thermal conductivity, namely, increase/decrease at low/high temperatures is remarkable. The existence of a maximum in the thermal conductivity can also be inferred form our simulation data. This represents to best of our knowledge the first time such behavior has been reported in simulations.

To analyse the accuracy of the simulation results against the experimental data we have

represented in Figure 6 the relative error, $\Delta \lambda=\frac{\lambda_{\text {sim. }}-\lambda_{\exp } .}{\lambda_{\exp .}}$, for each thermodynamic state, where $\lambda_{\text {exp. }}$ are data taken from reference ${ }^{31}$. We represent the error with reference to the phase coexistence diagram of water, in order to give a clearer view of the performance of the empirical models as a function of the thermodynamic state. Figures 6-a and b, clearly show larger differences with experimental data at high temperatures and at low and high densities. Interestingly, the models perform better for densities close to the coexistence one, with differences between simulation and experiment within $\sim 10 \%$. At high temperature and density the agreement is quantitative. At low temperatures, $T \sim 300 \mathrm{~K}$, the thermal conductivity is overestimated, between 20-30 \%. This results is interesting as the two models investigated in this work have been fitted to reproduce the properties of water at this temperature and the corresponding liquid density at 1 bar pressure. At high temperatures and densities lower than the critical one, the thermal conductivity is underestimated between $30-40 \%$.

\section{CONCLUSIONS}

We have employed boundary driven molecular dynamics simulations to investigate the accuracy of two of the most accurate rigid non polarizable models in predicting the thermal conductivity of water. An accurate prediction of this property for water encloses some challenges not found in other fluids, as water features the highest thermal conductivity of any molecular liquid. Moreover, following the behaviour observed in other water properties, the thermal conductivity features an anomalous response, namely, it increases with temperature at temperatures below 400-500 K, an it features a maximum. Beyond this maximum it decreases following the normal behavior observed in simple liquids.

It is remarkable that both $\mathrm{SPC} / \mathrm{E}$ and $\mathrm{TIP} 4 \mathrm{P} / 2005$ are able to reproduce this complex behaviour. At a quantitative level both models predict the correct order of magnitude of the 
thermal conductivity, as well is its dependence with pressure and temperature. The models are comparable in terms of accuracy. Interestingly, the introduction of a fourth site in the TIP4P/2005 model, which has been shown to play a key role in getting closer agreement with experiments, seems to play a minor role in determining the heat conduction.

Both SPC/E and TIP4P/2005 models are accurate in a well defined region of the phase diagram, corresponding to temperatures between $300 \mathrm{~K}$ and the critical temperature and densities close to the liquid coexistence line. We also find that the accuracy of the model is excellent at supercritical temperatures and densities higher than the critical one.

The thermal conductivity is a complex property. Its magnitude is the result of a complex interplay between the temperature and pressure dependence of the density, as well as the formation/breaking of the hydrogen bond network. The reorganization of the hydrogen bond structure is deemed to be an essential factor in determining the anomalous dependence of the thermal conductivity with temperature. Hence the thermal conductivity represents a stringent test to validate water models. Improving the agreement with experiment, particularly at low densities may require the introduction of polarizability, a factor that is missed in the rigid models investigated here.

Overall, our results show that rigid non polarizable models provide a good prediction of the thermal conductivity of water and its anomalous behavior in a wide range of thermodynamic conditions. This result is of particular significance, as it shows that rigid non polarizable models incorporate the main ingredients defining heat conduction in water, and therefore they provide a route to advance in our understanding of the microscopic mechanism determining heat transfer in water.

\section{ACKNOWLEDGMENTS}

We would like to thank the Leverhulme Trust for financial support and the Imperial College High Performance Computing Service for providing computational resources. We would like to thank Professor Jan Sengers for enlightening discussion and for providing reference ${ }^{31}$ before publication. FB would like to thank the EPSRC for support in the form of a Leadership Fellowship. 


\section{REFERENCES}

${ }^{1}$ W. T. Ashurst and W. G. Hoover, Phys. Rev. A 11, 658 (1975).

${ }^{2}$ B. Hafskjold and T. Ikeshoji and S. K. Ratkje, Mol. Phys. 80, 1389 (1993).

${ }^{3}$ F. Bresme, B. Hafskjold, and I. Wold, J. Phys. Chem. 100, 1879 (1996).

${ }^{4}$ D. Reith and F. Müller-Plathe, J. Chem. Phys. 112, 2436 (2000).

${ }^{5}$ A. Perronace, C. Leppla, F. Leroy, B. Rousseau, and S. Wiegand, J. Chem. Phys. 116, 3718 (2002).

${ }^{6}$ N. Galamba, C. N. de Castro, and J. Ely, J. Chem. Phys. 126, 204511 (2007).

${ }^{7}$ P. Keblinski, S. Phillpott, S. Choi, and J. Eastman, International Journal of Heat and Mass Transfer 45, 855 (2002).

${ }^{8}$ H. Patel, S. Das, T. Sundararajan, A. Nair, B. George, and T. Pradeepa, Appl. Phys. Lett. 83, 2931 (2003).

${ }^{9}$ A. Lervik, F. Bresme, and S. Kjelstrup, Soft Matter 5, 2407 (2009).

${ }^{10}$ D. Cahill, W. Ford, K. Goodson, G. Mahan, A. Majumdar, H. Maris, R. Merlin, and S. Phillpot, J. Appl. Phys. 93, 793 (2003).

${ }^{11}$ D. Leitner, Annual Review of Physical Chemistry 59, 233 (2009).

${ }^{12}$ A. Lervik, F. Bresme, S. Kjelstrup, D. Bedeaux, and J. Rubí, Phys. Chem. Chem. Phys. 12, $1610(2010)$.

${ }^{13}$ H. Berendsen, J. Grigera, and T. Straatsma, J. Phys. Chem. 91, 6269 (1987).

${ }^{14}$ J. L. F. Abascal and C. Vega, J. Chem. Phys. 123, 234505 (2005).

${ }^{15}$ C. Vega and J. Abascal, Phys. Chem. Chem. Phys. 13, 19663 (2011).

${ }^{16}$ J.L.F. Abascal and C. Vega, Phys. Rev. Lett. 98, 237801 (2007).

${ }^{17}$ D. Bedrov and G. Smith, J. Chem. Phys. 113, 8080 (2000).

${ }^{18}$ F. Bresme, J. Chem. Phys. 115, 7564 (2001).

${ }^{19}$ M. Zhang, E. Lussetti, L. Souza, and F. Müller-Plathe, J. Phys. Chem. B 109, 15060 (2005).

${ }^{20}$ W. Evans, J. Fish, and P. Keblinski, J. Chem. Phys. 126, 154504 (2007).

${ }^{21}$ J. Muscatello, F. Römer, J. Sala, and F. Bresme, Phys. Chem. Chem. Phys. 13, 19970 (2011).

${ }^{22}$ J. Muscatello and F. Bresme, J. Chem. Phys. 135, 234111 (2011).

${ }^{23}$ P. Kumar and H.E. Stanley, J. Phys. Chem. B 115, 14269 (2011). 
${ }^{24}$ R. W. Hockney, Methods Comput. Phys. 9, 136 (1970).

${ }^{25}$ U. Essmann, L. Perera, M. L. Berkowitz, T. Darden, H. Lee, and L. G. Pedersen, J. Chem. Phys. 103, 8577 (1995).

${ }^{26}$ S. Miyamoto and P. A. Kollman, J. Comp. Chem. 13, 952 (1992).

${ }^{27}$ G. Bussi, D. Donadio, and M. Parrinello, J. Chem. Phys. 126, 014101 (2007).

${ }^{28}$ B. Hess, C. Kutzner, D. van der Spoel, and E. Lindahl, J. Chem. Theor. Comp. 4, 435 (2008).

${ }^{29}$ National Institute of Standards and Technology, "Nist chemistry webbook," http://webbook.nist.gov/chemistry/.

${ }^{30}$ S. De Groot and P. Mazur, Non-equilibrium Thermodynamics, 1st ed. (North Holland Publishing Co., 1963).

${ }^{31}$ M. Huber, R. Perkin, D. Friend, J. Sengers, M. Assael, I. Metaxa, K. Miyagawa, R. Hellmann, and E. Vogel, J. Phys. Chem. Ref. Data, in press (2012). 


\section{TABLES}

TABLE I. Thermal conductivity data for the TIP4P/2005 model at different thermodynamics states. $\lambda_{\text {sim }}$ represents the thermal conductivity obtained from the simulations, and $\lambda_{\text {exp. }}$ corresponds to data obtained from reference ${ }^{31}$.

\begin{tabular}{ccccccccc}
\hline \hline$\frac{T_{\text {ave }}}{\mathrm{K}}$ & $\frac{\rho_{\text {ave }}}{\mathrm{g} \mathrm{cm}^{-3}}$ & $\frac{p}{\mathrm{bar}}$ & $\frac{\nabla T}{\mathrm{~K} \mathrm{~nm}}$ & $\frac{J_{U}}{10^{10} \mathrm{~W} \mathrm{~m}^{-2}}$ & $\frac{\lambda_{\text {sim }}}{\mathrm{W} \mathrm{m}^{-1} \mathrm{~K}^{-1}}$ & $\frac{\lambda_{\text {exp. }}}{\mathrm{W} \mathrm{m} \mathrm{m}^{-1} \mathrm{~K}^{-1}}$ & $\frac{\Delta \lambda}{\%}$ \\
\hline 324 & 1.0 & $393 \pm 23$ & $-19.00 \pm 0.02$ & $1.62 \pm 0.06$ & $0.85 \pm 0.03$ & 0.66 & 30 \\
424 & 1.0 & $2033 \pm 25$ & $-18.99 \pm 0.03$ & $1.77 \pm 0.08$ & $0.93 \pm 0.04$ & 0.80 & 17 \\
522 & 1.0 & $4140 \pm 27$ & $-18.78 \pm 0.05$ & $1.83 \pm 0.09$ & $0.98 \pm 0.05$ & 0.91 & 8 \\
623 & 1.0 & $6468 \pm 30$ & $-19.05 \pm 0.05$ & $1.93 \pm 0.10$ & $1.01 \pm 0.05$ & 1.00 & 1 \\
723 & 1.0 & $8722 \pm 35$ & $-18.45 \pm 0.05$ & $1.98 \pm 0.11$ & $1.07 \pm 0.06$ & 1.08 & $<1$ \\
521 & 0.9 & $1530 \pm 24$ & $-18.85 \pm 0.04$ & $1.53 \pm 0.08$ & $0.81 \pm 0.04$ & 0.74 & 10 \\
622 & 0.9 & $3313 \pm 27$ & $-19.15 \pm 0.05$ & $1.56 \pm 0.09$ & $0.82 \pm 0.05$ & 0.80 & 2 \\
721 & 0.9 & $5116 \pm 31$ & $-18.80 \pm 0.06$ & $1.62 \pm 0.09$ & $0.86 \pm 0.05$ & 0.86 & $<1$ \\
621 & 0.8 & $1512 \pm 24$ & $-19.07 \pm 0.06$ & $1.34 \pm 0.08$ & $0.71 \pm 0.04$ & 0.65 & 8 \\
720 & 0.8 & $2883 \pm 27$ & $-18.73 \pm 0.06$ & $1.38 \pm 0.09$ & $0.73 \pm 0.05$ & 0.69 & 6 \\
619 & 0.7 & $578 \pm 23$ & $-19.09 \pm 0.05$ & $1.12 \pm 0.07$ & $0.59 \pm 0.04$ & 0.55 & 8 \\
719 & 0.7 & $1599 \pm 25$ & $-18.55 \pm 0.05$ & $1.09 \pm 0.07$ & $0.59 \pm 0.04$ & 0.56 & 5 \\
718 & 0.6 & $942 \pm 22$ & $-18.05 \pm 0.07$ & $0.88 \pm 0.07$ & $0.49 \pm 0.04$ & 0.46 & 5 \\
713 & 0.5 & $573 \pm 17$ & $-18.19 \pm 0.07$ & $0.70 \pm 0.05$ & $0.38 \pm 0.03$ & 0.39 & -1 \\
704 & 0.4 & $366 \pm 15$ & $-17.12 \pm 0.11$ & $0.50 \pm 0.04$ & $0.29 \pm 0.03$ & 0.33 & -10 \\
688 & 0.3 & $240 \pm 11$ & $-16.16 \pm 0.19$ & $0.31 \pm 0.03$ & $0.19 \pm 0.02$ & 0.28 & -31 \\
\hline \hline
\end{tabular}


TABLE II. Thermal conductivity data for the SPC/E model.

\begin{tabular}{ccccccccc}
\hline \hline$\frac{T_{\text {ave }}}{\mathrm{K}}$ & $\frac{\rho_{\text {ave }}}{\mathrm{g} \mathrm{cm}^{-3}}$ & $\frac{p}{\mathrm{bar}}$ & $\frac{\nabla T}{\mathrm{~K} \mathrm{~nm}}$ & $\frac{J_{U}}{10^{10} \mathrm{~W} \mathrm{~m} \mathrm{~m}^{-2}}$ & $\frac{\lambda_{\text {sim. }}}{\mathrm{W} \mathrm{m}^{-1} \mathrm{~K}^{-1}}$ & $\frac{\lambda_{\text {exp. }}}{\mathrm{W} \mathrm{m} \mathrm{K}^{-1}}$ & $\frac{\Delta \lambda}{\%}$ \\
\hline 325 & 1.0 & $454 \pm 24$ & $-18.97 \pm 0.03$ & $1.66 \pm 0.07$ & $0.87 \pm 0.04$ & 0.66 & 33 \\
424 & 1.0 & $2139 \pm 27$ & $-18.68 \pm 0.03$ & $1.75 \pm 0.08$ & $0.94 \pm 0.04$ & 0.80 & 18 \\
525 & 1.0 & $4156 \pm 29$ & $-18.94 \pm 0.03$ & $1.87 \pm 0.09$ & $0.98 \pm 0.05$ & 0.91 & 8 \\
626 & 1.0 & $6378 \pm 30$ & $-18.70 \pm 0.05$ & $1.85 \pm 0.10$ & $0.99 \pm 0.05$ & 1.00 & -1 \\
726 & 1.0 & $8549 \pm 36$ & $-19.09 \pm 0.05$ & $1.94 \pm 0.10$ & $1.02 \pm 0.05$ & 1.08 & -6 \\
524 & 0.9 & $1712 \pm 26$ & $-18.45 \pm 0.05$ & $1.54 \pm 0.08$ & $0.84 \pm 0.04$ & 0.74 & 13 \\
625 & 0.9 & $3432 \pm 27$ & $-19.35 \pm 0.06$ & $1.56 \pm 0.08$ & $0.81 \pm 0.04$ & 0.80 & $<1$ \\
725 & 0.9 & $5127 \pm 30$ & $-19.16 \pm 0.07$ & $1.60 \pm 0.09$ & $0.84 \pm 0.05$ & 0.87 & -4 \\
624 & 0.8 & $1639 \pm 24$ & $-19.04 \pm 0.05$ & $1.28 \pm 0.08$ & $0.67 \pm 0.04$ & 0.65 & 3 \\
724 & 0.8 & $3016 \pm 26$ & $-19.29 \pm 0.05$ & $1.37 \pm 0.08$ & $0.71 \pm 0.04$ & 0.69 & 3 \\
623 & 0.7 & $738 \pm 22$ & $-18.47 \pm 0.06$ & $1.08 \pm 0.07$ & $0.58 \pm 0.04$ & 0.55 & 7 \\
722 & 0.7 & $1728 \pm 23$ & $-18.27 \pm 0.07$ & $1.08 \pm 0.07$ & $0.59 \pm 0.04$ & 0.56 & 5 \\
721 & 0.6 & $1041 \pm 21$ & $-18.60 \pm 0.06$ & $0.88 \pm 0.07$ & $0.47 \pm 0.04$ & 0.46 & 2 \\
716 & 0.5 & $632 \pm 17$ & $-18.46 \pm 0.08$ & $0.69 \pm 0.05$ & $0.37 \pm 0.03$ & 0.39 & -3 \\
711 & 0.4 & $428 \pm 15$ & $-17.30 \pm 0.09$ & $0.52 \pm 0.04$ & $0.30 \pm 0.02$ & 0.32 & -7 \\
700 & 0.3 & $294 \pm 10$ & $-16.29 \pm 0.14$ & $0.35 \pm 0.03$ & $0.21 \pm 0.02$ & 0.26 & -19 \\
\hline \hline & 0.2 & $187 \pm 8$ & $-16.53 \pm 0.13$ & $0.21 \pm 0.02$ & $0.13 \pm 0.01$ & 0.19 & -33 \\
\hline
\end{tabular}

